\title{
Estudo da Incorporação da Borra Oleosa de Petróleo em Materiais Estabilizados por Solidificação
}

\author{
Maria Rosiane de Almeida Andrade* (Mestranda em Engenharia Química na Universidade Federal de Campina \\ Grande); \\ Deborah Almeida dos Anjos (Mestranda em Engenharia Química na Universidade Federal de Campina Grande); \\ Márcia Cristina de Sousa (Graduanda em Engenharia Química na Universidade Federal de Campina Grande); \\ Maria Isabel do Bú Araújo (Graduanda em Engenharia Química na Universidade Federal de Campina Grande); \\ André Luiz Fiquene de Brito (Prof. da UAEQ na Universidade Federal de campina Grande).
}

\section{Resumo:}

Indústrias de petróleo são reconhecidas mundialmente como uma atividade com grandes impactos ambientais. Um dos resíduos gerados é a borra oleosa de petróleo, produzida em refinarias, que contém óleos e graxas, metais pesados e hidrocarbonetos totais. Assim, se faz necessário um tratamento da borra oleosa de petróleo antes de sua disposição final. Este trabalho tem como objetivo realizar o tratamento da borra oleosa utilizando a estabilização por solidificação, técnica que restringe a mobilidade dos contaminantes. $\mathrm{O}$ trabalho foi dividido em 3 etapas: planejamento experimental, preparação dos corpos de prova e avaliação do material. Foi adotado o planejamento experimental fatorial $2^{2}$ onde os fatores adotados foram: A - Porcentagem de borra oleosa e B - Tempo de cura. Para o fator A, porcentagem de resíduo, foram usados os níveis 10 e $20 \%$ codificados para baixo (-) e alto $(+)$, respectivamente. Para o fator $B$, tempo de cura, foram usados níveis 14 e 28 dias, codificados nos níveis baixo $(-)$ e alto $(+)$. Os corpos de prova foram preparados tendo como aglomerante o cimento Portland comum e cal. As matrizes foram avaliadas através dos ensaios de integridade e durabilidade, sendo estes, resistência à compressão e capacidade de absorção de água. Observou-se que para a resistência à compressão apenas a porcentagem da borra oleosa influencia na resposta e que para a capacidade de absorção de água a porcentagem da borra e a interação entre o tempo de cura e a porcentagem de resíduo influenciam na resposta. Todos os materiais apresentaram um valor de resistência à compressão menor que $1 \mathrm{MPa}$, logo o material deve ser disposto em aterros de resíduos não-perigosos. Quanto à capacidade de absorção de água, o material apresentou valores abaixo de $40 \%$ que é o limite máximo permissível. 


\section{I ntrodução}

Refinarias de petróleo e indústrias petroquímicas geram grandes quantidades de resíduos sólidos. Uma preocupação especial é a borra oleosa que se acumula no fundo dos tanques de armazenagem de petróleo bruto ou é gerada em sistemas de separação de água-óleo (KRIIPSALU, MARQUES, e MAASTIK, 2008). Estimativas indicam que uma indústria petroquímica que processa 200 barris de petróleo por dia gera cerca de $10.000 \mathrm{~m}^{3}$ de borra oleosa de petróleo (GAFAROV et al, 2006).

A borra oleosa de petróleo contém grandes quantidades de alcanos, cicloalcanos, benzeno, tolueno, xilenos, fenóis e hidrocarbonetos aromáticos policíclicos (HPAs), metais pesados e elevado teor de óleos e graxas. Muitos componentes da lama oleosa são listados como poluentes prioritários pela Agência de Proteção Ambiental dos EUA, devido à sua toxicidade e seu potencial mutagênico e carcinogênico para os seres humanos (DHOTE et al, 2006; JANBANDHU e FULEKAR, 2011). A ABNT NBR 10004 (2004) classifica a borra oleosa de petróleo como um resíduo perigoso Classe I. Logo, se faz necessário desenvolver técnicas de tratamento para a borra oleosa de petróleo.

A Estabilização por Solidificação (E/S) é uma técnica que vem sendo utilizada a mais de cinquenta anos no tratamento de resíduos que não podem ser reciclados, reduzidos ou reutilizados. O principal objetivo da E/S é reduzir a mobilidade dos contaminantes por lixiviação.

Catalan (2002) diz que a E/S apresenta como objetivos: “imobilizar os contaminantes através de mecanismos químicos e físicos, a fim de reduzir a velocidade em que os contaminantes podem ser transportados para o meio ambiente; aumentar a integridade estrutural do material tratado".

O processo de E/S envolve a mistura de resíduo tanto na forma de lodo como líquido e sólido, em um material cimentício, de forma a encapsular e incorporar o resíduo nesse sistema de ligação tendo um material sólido com integridade estrutural e estabilidade para haver o mínimo de resíduo lixiviado do sistema (FITCH e CHEESEMAN, 2003).

$\mathrm{O}$ tratamento por E/S combina dois processos inter-relacionados que ocorrem simultaneamente, para se obter um produto que terá menor impacto ambiental quando descartado ou reutilizado. A solidificação é o processo de produzir um produto sólido, com propriedades físicas melhoradas (principalmente de resistência), e estabilização é o processo de converter o contaminante para uma forma menos móvel e menos tóxico (CONNER e HOEFFNER, 1998; YILMAZ, UNLU e COKCA, 2003).

Neste contexto, o presente trabalho teve como objetivo realizar o tratamento da borra oleosa de petróleo utilizando a estabilização por solidificação e tendo como ligantes o cimento Portland comum e o hidróxido de cálcio.

\section{Materiais e métodos}

\subsection{Planejamento de experimento e análise estatística}

Foi adotado o planejamento experimental fatorial $2^{2}$. Os fatores adotados foram: A - Tempo de cura e B - Porcentagem de borra oleosa. O experimento teve como objetivo verificar se existe efeito significativo entre a porcentagem de borra oleosa e o tempo de cura, além das interações entre estes fatores, através da análise de variância - ANOVA. O critério de decisão a ser adotado será o seguinte:

- Efeito Significativo (valor $\mathrm{p} \leq 0,05$ ): Médias diferentes ao nível de $95 \%$ de Probabilidade;

- Efeito Não Significativo (valor p > 0,05): Médias iguais ao nível de 95\% de Probabilidade.

\subsection{Caracterização da borra oleosa de petróleo e dos aglomerantes}

A borra oleosa de petróleo, o cimento e a cal foram caracterizados determinando os sólidos e suas frações presentes.

Pesou-se 25 gramas de cada material (resíduo e aglomerantes) separadamente e enviou para estufa por 24 horas à temperatura de $103-105{ }^{\circ} \mathrm{C}$, obtendo-se o material seco. Retiraram-se os materiais, deixou-os esfriar no dessecador e pesou novamente. Em seguida, os materiais foram levados para incinerar à temperatura de $550{ }^{\circ} \mathrm{C}$ no forno mufla por 1 hora (resíduo calcinado). As amostras foram esfriadas no dessecador, e então as pesou novamente. 
(2) e (3).

Os resultados para sólidos totais, fixos e voláteis, foram calculados através das equações (1),

$$
\begin{aligned}
& \text { SólidosTotais }(\%)=\left[\frac{(A-B)}{(C-B)}\right] * 100 \\
& \text { SólidosVoláteis }(\%)=\left[\frac{(A-D)}{(A-B)}\right] * 100 \\
& \text { SólidosFixos }(\%)=\left[\frac{(D-B)}{(A-B)}\right] * 100
\end{aligned}
$$

Onde: A: peso da amostra seca + cápsula; B : peso da cápsula; C : peso da amostra úmida + cápsula; D : peso do resíduo calcinado + cápsula.

\subsection{Preparação dos corpos de prova}

Os corpos de provas foram preparados seguindo as etapas propostas pela ABNT NBR 7215 (1996) e utilizando o Protocolo de avaliação de materiais E/S (BRITO, 2007). O aglomerante utilizado foi uma mistura de cimento Portland e hidróxido de cálcio e os fatores adotados foram: porcentagem de borra oleosa de petróleo (10 e 20\%) e tempo de cura (14 e 28 dias).

Inicialmente misturou-se o aglomerante com a borra oleosa de petróleo, em seguida adicionouse água aquecida a $60{ }^{\circ} \mathrm{C}$. A partir do contato entre os aglomerantes com água, iniciou-se a contagem do tempo de preparação dos corpos de provas. Homogeneizou-se bem a mistura de forma a obter uma massa homogênea. A massa formada foi então disposta em moldes cilíndricos e foram cobertos com placas de vidros afim de não ocorrer perda d'água. Os corpos de prova ficaram em repouso por um período de 24 horas para endurecimento da pasta. Após as 24h, os corpos de prova foram desmoldados e deixados por um tempo de cura de 14 e 28 dias, para que então os ensaios fossem realizados.

\subsection{Resistência à compressão}

No ensaio de Resistência à Compressão (RC), foram utilizados corpos de prova cilíndricos de $50 \mathrm{~mm}$ de diâmetro e $100 \mathrm{~mm}$ de altura, os quais foram postos diretamente sobre o prato inferior de uma prensa, de maneira que ficassem rigorosamente centrados em relação ao eixo de carregamento. A medida da resistência à compressão foi calculada pela Expressão (4), em kgf.cm², considerando a carga aplicada (F) e a área da seção do corpo de prova (A), e convertida para MPa.

$\mathrm{RC}\left(\mathrm{kgf} . \mathrm{cm}^{2}\right)=\mathrm{F} / \mathrm{A}$

\subsection{Capacidade de absorção de água}

No ensaio de capacidade de absorção de água os corpos de provas foram condicionados em estufa a $103{ }^{\circ} \mathrm{C}$ por 24 horas e com uma relação líquido/sólido (L/S) 10:1, utilizando água destilada. Posteriormente as amostras foram imersas em água a $23{ }^{\circ} \mathrm{C}$ por períodos de 24 , 48 e 72 horas. O resultado é expresso em \% conhecendo-se a massa do corpo de prova após saturação em água e a massa do corpo de prova seca em estufa, conforme a expressão (5)

$$
C A A(\%)=\frac{M S A T-M S}{M S} \times 100
$$

Onde: MSAT: massa do corpo de prova após saturação em água e fervura; MS: massa do corpo de prova seco em estufa.

\section{Resultados e discussão}




\subsection{Caracterização da borra oleosa de petróleo e dos aglomerantes}

Na Tabela 1 estão apresentados os valores de umidade, dos sólidos e suas frações e do pH da borra oleosa, do cimento e da cal.

Tabela 1- Valores de umidade, dos sólidos e suas frações e pH da borra oleosa

\begin{tabular}{|c|c|c|c|}
\hline Parâmetros & Borra (\%) & Cal & Cimento \\
\hline Teor de umidade & 15,04 & 5,41 & 0,183 \\
\hline Sólidos totais & 84,96 & 95,52 & 99,817 \\
\hline Sólidos totais fixos & 57,00 & 82,82 & 98,465 \\
\hline Sólidos totais voláteis & 43,30 & 17,13 & 1,535 \\
\hline $\mathrm{pH}$ & 7,5 & & \\
\hline
\end{tabular}

O teor de sólidos voláteis apresentado na borra oleosa (43,30\%) indica a fração de compostos orgânicos, que no caso da borra de petróleo é representado pelos hidrocarbonetos voláteis tais como o benzeno, tolueno, etilbenzeno, xileno (BTEX) e outros hidrocarbonetos facilmente volatilizáveis à temperatura de $550^{\circ} \mathrm{C}$. O elevado teor de sólidos fixos (57\%) sugere a presença de materiais inertes, como areia e frações de argila, comuns neste tipo de resíduo (OLIVEIRA, 2003).

Os resultados mostram que o cimento apresenta concentrações elevadas de sólidos totais e fixos. O que significa que este tem menores concentrações de compostos que se volatilizam a temperaturas menores que $550{ }^{\circ} \mathrm{C}$, ou seja, tem teores menores de matéria orgânica, quando comparado com a cal.

\subsection{Resistência à compressão}

Na Tabela 2 estão apresentados os valores obtidos para o ensaio de resistência à compressão (RC).

Tabela 2 - Resultados de resistência à compressão

\begin{tabular}{cccc}
\hline Experimento & Tempo de cura (dias) & Borra oleosa (\%) & RC(Mpa( \\
\hline 1 & 14 & 10 & 0,90 \\
2 & 28 & 10 & 0,50 \\
3 & 14 & 20 & 0,39 \\
4 & 28 & 20 & 0,15 \\
5 & 14 & 10 & 0,80 \\
6 & 28 & 10 & 0,70 \\
7 & 14 & 20 & 0,53 \\
8 & 28 & 20 & 0,37 \\
\hline
\end{tabular}

O valor mínimo estabelecido pelo Protocolo de Avaliação de Materiais Estabilizados por Solidificação proposto por Brito (2007) para o resultado de resistência à compressão é de 1 MPa, logo a partir dos dados apresentados na Tabela 2 percebe-se que todos os tratamentos ficaram abaixo do limite mínimo.

Segundo Brito (2007) se o material apresentar resistência à compressão menor que $1 \mathrm{MPa}$, sua utilização será controlada e dispostas em aterro de resíduos não perigosos. Neste caso a concentração do contaminante deve ser quantificada para decidir a rota final a ser seguida.

A Tabela 3 mostra a ANOVA para a resistência a compressão.

Tabela 3. Análise de Variância (ANOVA) para RC

\begin{tabular}{ccccc}
\hline Fontes de Variação & G.L & $\begin{array}{c}\text { Soma Quadrática } \\
(\text { SQ) }\end{array}$ & $\begin{array}{c}\text { Quadrado Médio Ajustado } \\
\text { (QMaj) }\end{array}$ & p-valor * \\
\hline Efeito Principal & 2 & 0,37 & 0,18 & 0,019 \\
A - Fator tempo(t) & 1 & 0,10 & 0,10 & 0,059 \\
B - Fator porc (\%) & 1 & 0,27 & 0,27 & 0,013 \\
\hline
\end{tabular}


Tabela 3. Análise de Variância (ANOVA) para RC

\begin{tabular}{ccccc} 
& & & & (Continuação) \\
\hline AB - Interação porc $x$ & 1 & 0,001 & 0,001 & 0,785 \\
tempo & 4 & 0,06 & 0,01 & \\
Residual & 4 & 0,06 & 0,01 & \\
Puro Erro & 7 & 0,43 & & \\
Total & $\mathrm{R}^{2} \mathrm{max}=$ & & & \\
$\mathrm{R}^{2}=86,21$ & 75,87 & &
\end{tabular}

LEGENDA: G.L: Graus de liberdade; R2: Coeficiente de Determinação;

A Tabela 3 mostra que apenas o fator porcentagem de borra oleosa de petróleo é significativo ao nível de $95 \%$ de probabilidade, pois apenas este valor p foi menor que 0,05 . Logo, é valido afirmar que apenas a porcentagem influência na resistência a compressão.

A Figura 1 confirma o resultado da ANOVA apresentada na tabela anterior, pois, apresenta o gráfico de Pareto para os efeitos padronizados para a resistência à compressão ao nível de $95 \%$ de probabilidade. Observa-se que só o fator porcentagem (B) é interceptado pela linha dos efeitos padronizados.

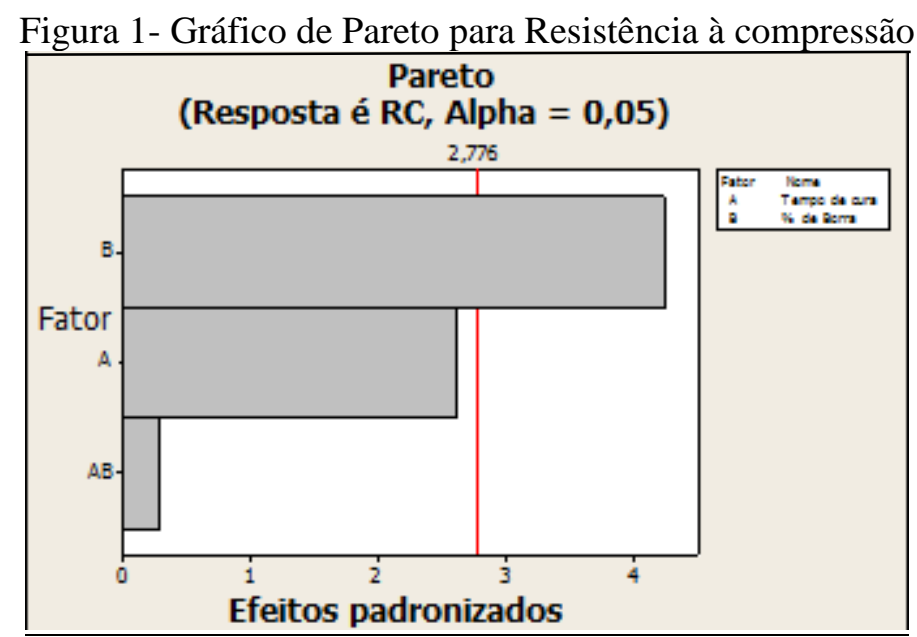

O modelo obtido está apresentado na Expressão (6). O modelo consegue explicar 86,21\% de um máximo de 75,87\%.

$\mathrm{RC}=0,54-018$ porc

\subsection{Capacidade de absorção de água}

Na Tabela 4 estão apresentados os valores obtidos para o ensaio de capacidade de absorção de água (CAA).

Tabela 4 - Resultados capacidade de absorção de água

\begin{tabular}{cccc}
\hline Experimento & Tempo de cura (dias) & Borra oleosa (\%) & CAA \\
\hline 1 & 14 & 10 & 26,78 \\
2 & 28 & 10 & 22,95 \\
3 & 14 & 20 & 21,43 \\
4 & 28 & 20 & 24,61 \\
5 & 14 & 10 & 26,55 \\
6 & 28 & 10 & 23,26 \\
7 & 14 & 20 & 22,32 \\
8 & 28 & 20 & 25,00 \\
\hline
\end{tabular}


Segundo a ABNT NBR 9778 (1987), no ensaio de capacidade de absorção de água o limite máximo de água permissível nos poros é 40\%. Pelos dados apresentados na Tabela 3, todos os ensaios foram aprovados neste ensaio.

A Tabela 5 mostra a ANOVA para a capacidade de absorção de água. Tabela 5. Análise de Variância (ANOVA) para CAA

\begin{tabular}{ccccc}
\hline Fontes de Variação & G.L & $\begin{array}{c}\text { Soma Quadrática } \\
\text { (SQ) }\end{array}$ & $\begin{array}{c}\text { Quadrado Médio Ajustado } \\
\text { (QMaj) }\end{array}$ & p-valor * \\
\hline Efeito Principal & 2 & 4,97 & 2,49 & 0,01 \\
A - Fator tempo( t) & 1 & 0,2 & 0,2 & 0,3 \\
B - Fator porc (\%) & 1 & 4,77 & 4,77 & 0,004 \\
AB - Interação porc x & 1 & 21,06 & 21,06 & 0,0 \\
tempo & & & & \\
Residual & 4 & 0,55 & 0,14 & \\
Puro Erro & 4 & 0,55 & & \\
Total & 7 & 26,58 & & \\
$\mathrm{R}^{2}=97,94$ & $\mathrm{R}^{2} \max =$ & & & \\
& 96,40 & & & \\
\hline
\end{tabular}

LEGENDA: G.L: Graus de liberdade; R2: Coeficiente de Determinação;

A partir dos dados apresentados na Tabela 4 percebe-se que apenas o fator porcentagem de borra oleosa e a interação entre a porcentagem de borra e o tempo de cura influenciam na capacidade de absorção de água, pois só para estes dois parâmetros que o valor de p foi menor que 0,05.

A Figura 2 apresenta o gráfico de Pareto para os efeitos padronizados para a capacidade de absorção de água ao nível de 95\% de probabilidade. Observa-se que o fator porcentagem (B) e a interação (AB) são interceptados pela linha dos efeitos padronizados.

Figura 2- Gráfico de Pareto para capacidade de absorção de água

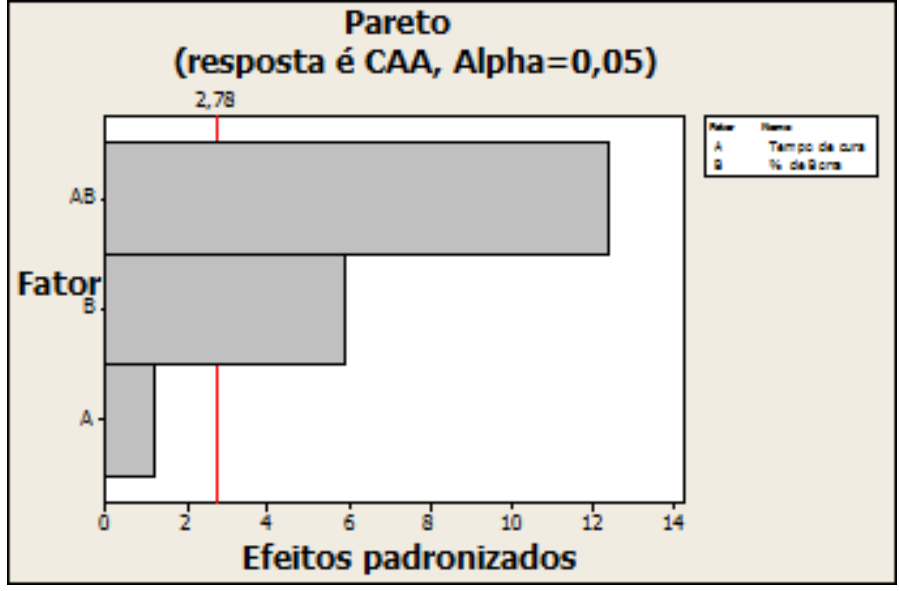

O modelo obtido está apresentado na Expressão (7). O modelo consegue explicar 97,94\% de um máximo de 96,4\%.

$\mathrm{CAA}=24,11-1,55$ porc $+3,25$ porc $\mathrm{x}$ tempo

\section{Conclusões}

Com base nos resultados apresentados, pode-se concluir que;

- A borra oleosa de petróleo apresentou um elevado teor de sólidos voláteis, o que indica a presença significativa de compostos orgânicos e o alto teor de sólidos totais pode ser um indicativo da presença de materiais inertes; 
- O alto teor de sólidos totais fixos no cimento indica a presença de materiais inertes, como areia e frações inorgânicas. E o elevado teor de sólidos voláteis na cal pode ser atribuído a presença de material orgânico;

- O cimento apresentou menor teor de umidade. Logo, pode-se dizer que o cimento tem facilidade de reagir com a água onde ocorre a sua hidratação rapidamente;

- Todos os tratamentos foram reprovados no ensaio de resistência à compressão e a partir do planejamento experimental pode-se dizer que apenas o fator porcentagem de borra oleosa influência na resistência à compressão;

- Todos os tratamentos foram aprovados no ensaio de capacidade de absorção de água e para esse ensaio a porcentagem de borra oleosa e a interação entre percentagem de borra e tempo de cura são os que influenciam na reposta. 


\title{
Study of Incorporation of Oily Sludge of Petroleum in Materials Stabilized by
}

\author{
Solidification
}

\begin{abstract}
Albstract: Petroleum industries are recognized worldwide as an activity with major environmental impacts. One of the waste generated is the oily sludge of petroleum, produced in refineries, which contains oil and grease, heavy metals and total hydrocarbons. Thus, treatment of sludge before its disposal is necessary. This work aims to conduct the treatment of oily sludge using the technique stabilization by solidification that restricts the mobility of contaminants. The work was divided into 3 stages: experimental planning, preparation of proof bodies and evaluation of the material. Was adopted factorial design $2^{2}$ where the adopted factors were: A - Percentage of oily sludge and B Curing time. For factor A, percentage of residue levels were used $10 \%$ and 20 encoded down $(-)$ and high $(+)$, respectively. For factor B, curing time, levels were used 14 and 28 days, the encoded low levels (-) and high (+).The samples were prepared having as binder ordinary Portland cement and lime. The matrices were evaluated using the integrity and durability tests, these being, compressive strength and capacity to absorb water. It was observed that resistance to compression only the percentage of oily sludge influences the response and the water absorption capacity of the percentage of the sludge and the interaction between the curing time and the residual percentage influence on the response. All the material had a resistance value to less compression than $1 \mathrm{MPa}$, then the material must be disposed of in non-hazardous waste landfills. Regarding water absorption capacity, the material showed values below $40 \%$ which is the maximum allowable limit. .
\end{abstract}

Keywordls: Stabilization; Solidification; Sludge oily. 


\section{Referências bibliográficas}

ASSOCIAÇÃO BRASILEIRA DE NORMAS TÉCNICAS - ABNT NBR 10.004: Resíduos Sólidos Classificação. CENW in, Versão Digital, ABNT NBR 10.004, 71p, $2004 \mathrm{a}$.

ASSOCIAÇÃO BRASILEIRA DE NORMAS TÉCNICAS - ABNT NBR 7.215: Determinação da resistência à compressão. Rio de Janeiro, 8p, 1996.

ASSOCIAÇÃO BRASILEIRA DE NORMAS TÉCNICAS - NBR 9.778: Argamassa e concreto endurecido Determinação da absorção de água por imersão. Rio de Janeiro, 5p, 1987.

BRITO, A. L. F. Protocolo de Avaliação de Materiais Resultantes da Estabilização por Solidificação. Doutorado em Engenharia Ambiental, Universidade Federal de Santa Catarina, 179: 2007.

CATALAN, L. J.J, et. al. Study of the physical and chemical mechanisms influencing the long-term environmental stability of natrojarosite waste treated by stabilization/solidification. In: J. Hazard. Mater. Elsevier, p. 63-88, 2002.

CONNER, J. R.; HOEFFNER, S. L. A critical review of stabilization/solidification technology. Crit. Rev. Environ. Sci. Technol. 28, p. 397-462, 1998.

DHOTE, M.; JUWARKAR, A.; KUMAR, A.; KANADE, G. S.; CHAKRABARTI, T.; Biodegradation of chrysene by the bacterial strains isolated from oily sludge. World J. Microbiol Biotechnol. 26, p. 329-335, 2009.

FITCH, J.R.; CHEESEMAN, C.R. Characterization of environmentally exposed cement based stabilized/solidified industrial waste. Journal of Hazardous Materials A, v. 101, n. 3, p. 239-255, 2003.

GAFAROV, A. B.; PANOV, A. V.; FILONOV, A. E.; BORONIN, A. M. Change in the composition of a bacterial association degrading aromatic compounds during oil sludge detoxification in a continuous-flow microbial reactor. Applied Biochemistry and Microbiology, New York, v. 42, n. 2, p. 160-165, 2006.

JANBANDHU, A.; FULEKAR, M. H.; Biodegradation of phenanthrene using adapted microbial consortium isolated from petrochemical contaminated environment. J. Hazard. Mater. 187, p. 333-340, 2011.

KRIIPSALU, M.; MARQUES, M.; MAASTIK, A.; Characterization of oily sludge from a wastewater treatment plant flocculation-flotation unit in a petroleum refinery and its treatment implications. J. Mater. Cycles Waste Manag. 10, p. 79-86, 2008.

OLIVEIRA, D. M. Aplicação da técnica de solubilização/estabilização para resíduos oleosos da indústria petrolífera, utilizando solo argiloso e bentonita. Mestrado em Engenharia Ambiental, Universidade de Santa Catarina, 139: 2003.

YILMAZ, O.; UNLU, K.; COKCA, E. Solidification/stabilization of hazardous wastes containing metals and organic contaminants. J. Environ. Eng. 129, p. 366-376, 2003. 